\section{Transparent ultra-water- repellent surfaces}

The clarity of optical surfaces, such as windows, often marred in wet conditions because of light refraction from the wetting film. Making such surfaces water-repellent - causing moisture to bead-up and form water droplets that simply roll off - provides a solution to this problem. Increasing a surface's water repellency can be eyeglasses and displays, is decreasing the energy

between it and water, and increasing its roughness. Although both are relatively straightforward, increasing a surface's roughness usually also reduces its optical clarity. But now Teshima et al. have developed a way to hydrophobize a rough surface of poly(ethylene terephthalate) (PET), while maintaining a high achieved by both

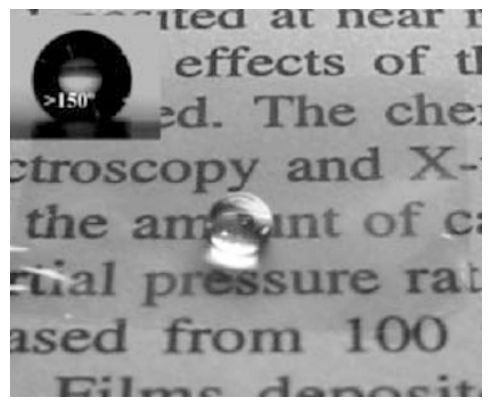

Water droplets on a transparent and ultra-waterrepellent PET substrate.

\section{Reprinted with} permission from ref. 1 Copyright 2003 American Chemical Society. transparency (Langmuir

http://dx.doi.org/10.1021/

1a034265d). The PET

surface was first treated using oxygen plasma, in

which non-crystalline regions were preferentially etched. The energy of this surface was then reduced by coating it with a layer of fluoroalkylsilane by chemical vapour deposition - the fluorine providing low energy due to its small radius and high electronegativity. This caused almost spherical water droplets to form on the surface with a contact angle of greater than $150^{\circ}$ (see image). A key aspect of the technique is that it is a low-temperature process, allowing polymeric substrates to be treated without thermal degradation.

\section{Elastomers with good memory}

Shape memory has been achieved in soft materials and at near-body temperature, which may allow for actuation in biomedical devices. Elastomers containing rod-shaped groups can form liquidcrystalline phases. When these groups are part of the main backbone of the polymer, the materials can exhibit a thermally stimulated movement, due to the combination of liquid-crystalline order and strain induced in the crosslinked network. However, the high (around $100^{\circ} \mathrm{C}$ ) transition temperature - from the ordered (liquid crystalline) to the disordered phase - has so far limited the use of this effect. Now researchers at the University of Connecticut have synthesized a liquid-crystalline elastomer having two different rod-shaped groups (I. A. Rousseau \& P. T. Mather, J. Am. Chem. Soc. http:dx.doi.org/10.1021/ja039001s). By varying the relative proportion of these groups they are able to tailor not only the transition temperatures, but also the mechanical properties of the material. A remarkable shape-memory effect was observed by stretching this material above its transition temperature and then cooling it to fix the new shape. The original shape could be easily recovered by heating again through the transition temperature, which was lower and close to body temperature.

\section{ION TRANSPORT IN NANOTUBES}

The detection and manipulation of single biomolecules is a thriving area of research with potential diagnostic applications. To achieve this goal, however, it is critical to get a better understanding of the ionic transport mechanisms arising from the passage of biomolecules through nanoscale channels. As reported in Nano Letters (http://dx.doi.org/10.1021/nl0348185), Hirofumi Daiguji and colleagues have developed a model to design inorganic nanotubes with the aim of controlling ion transport, and then clarify the effects of biomolecules on ion current, similar to that occurring in protein nanopores such as alpha-hemolysin. Their theoretical model suggest that when the diameter of the tube is smaller than the Debye length - the distance over which the local electric field affects the distribution of free charge carriers - a unipolar solution of counterions is created and co-ions are repelled from the channel. Therefore, by controlling the charge density in a region along the length of the channel, the ionic current can be modulated. It is proposed that this principle could form the basis of a unipolar ionic field-effect transistor device that could be used for weak-signal amplification and biomolecular sensing applications.

\title{
WORM memory
}

The advent of organic semiconductors promises to usher in a new age of low-cost consumer electronic devices. Already these materials have been used to make high-performance displays for mobile phones and other handheld devices, and many more applications from electronic paper to smart textiles could soon be brought to commercial reality. But although most of the components used in modern electronic circuitry such as transistors, logic gates and light-emitting devices have been demonstrated using organic semiconductors, surprisingly little attention has been paid to the one component that makes the information age possible, memory. Stephen Forrest and colleagues now report in Nature $(426,166-169 ; 2003)$ on an organic memory device that could help fill part of this gap. The device is a write-once read-many, or WORM, memory cell based on the common electrochromic polymer PEDOT. When a high enough voltage is applied to the cell, it undergoes a chemical reaction that irreversibly switches it from a conducting to an insulating state. The authors believe their WORM memories could provide a robust, low-power, low-cost alternative to conventional archival storage technologies such as CDs and magnetic hard-disk drives.

\section{Microhypodermics}

Hypodermic needles provide a cheap and efficient means for delivering drugs directly into the blood stream and other living tissues. But, as many drug molecules are just a few tens of nanometres or less in size, the millimetre bore of a conventional needle is many orders of magnitude larger than is required to achieve efficient delivery, causing unnecessary pain and limiting the ability to target specific tissues. With the aid of microfabrication techniques developed by the electronics industry, Devin McAllister and colleagues evaluate the use of arrays of micrometresized needles made from silicon, glass, metal and polymer to achieve targeted drug delivery through skin with minimal damage or discomfort (Proc. Natl Acad. Sci. 10,1375513760;2003). They find that arrays of solid silicon microneedles (each $150 \mu \mathrm{m}$ long and tapered from 80 to $1 \mu \mathrm{m}$ wide) increase the transport of particles of up to $50 \mathrm{~nm}$ in size through human epidermis by several orders of magnitude. Using arrays of hollow glass microneedles they were also able to administer insulin to diabetic rats to reduce the levels of glucose in their blood by around $70 \%$.

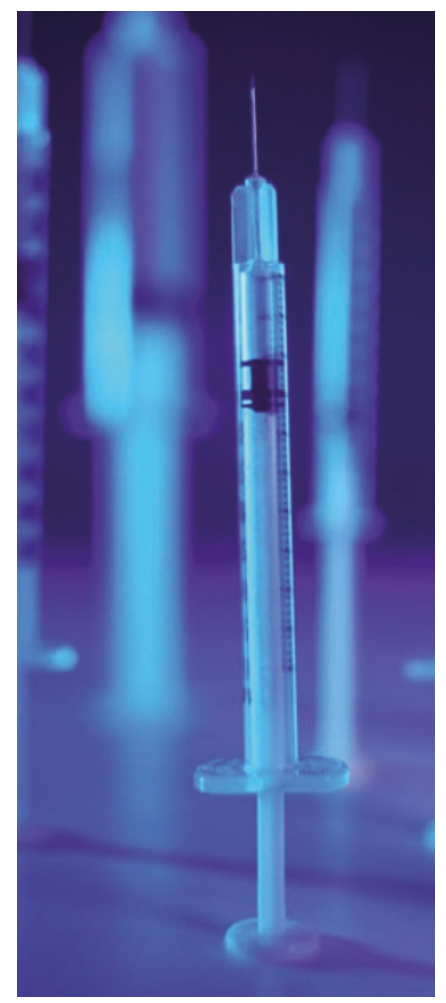

